\title{
An Expansin-Like Candidate Effector Protein from Pratylenchus penetrans Modulates Immune Responses in Nicotiana benthamiana
}

\author{
Paulo Vieira ${ }^{1,2}$ and Lev G. Nemchinov ${ }^{1, \dagger}$ \\ ${ }^{1}$ Molecular Plant Pathology Laboratory, U.S. Department of Agriculture-Agricultural Research Service, Beltsville, MD $20705-2350$ \\ ${ }^{2}$ School of Plant and Environmental Science, Virginia Tech, Blacksburg, VA 24061 \\ Accepted for publication 31 October 2019.
}

\begin{abstract}
The root lesion nematode (RLN) Pratylenchus penetrans is a migratory species that attacks a broad range of crops. After the RLN is initially attracted to host roots by root exudates and compounds, it releases secretions that are critical for successful parasitism. Among those secretions are nematode virulence factors or effectors that facilitate the entry and migration of nematodes through the roots and modulate plant immune defenses. The recognition of the effectors by host resistance proteins leads to effector-triggered immunity and incompatible plantnematode interactions. Although many candidate effectors of the RLN and other plant-parasitic nematodes have been identified, the detailed mechanisms of their functions and particularly, their host targets remain largely unexplored. In this study, we sequenced and annotated genes encoding expansin-like proteins, which are major candidate effectors of
\end{abstract}

ABSTRACT
$P$. penetrans. One of the genes, $P p-E X P B 1$, which was the most highly expressed during nematode infection in different plant species, was further functionally characterized via transient expression in the model plant Nicotiana benthamiana and global transcriptome profiling of gene expression changes triggered by this candidate effector in plants. As a result of this investigation, the biological roles of Pp-EXPB1 in nematode parasitism were proposed, the putative cellular targets of the proteins were identified, and the molecular mechanisms of plant responses to the nematode-secreted proteins were outlined.

Keywords: expansin-like candidate effector, expression profiling, nematology, Nicotiana benthamiana, Pratylenchus penetrans, transient expression
Root lesion nematodes (RLNs, Pratylenchus spp.) are economically important pathogens that inflict significant damage and yield losses on a wide range of crops (Castillo and Vovlas 2007; Vieira et al. 2018). This group of nematodes is listed third among the top 10 most economically important plant-parasitic nematodes (PPNs) (Jones et al. 2013). Pratylenchus spp. are migratory endoparasitic nematodes, and their mobility throughout their lifecycle causes extensive damage to the root system, predisposing the roots to secondary infections by other soilborne pathogens (Castillo and Vovlas 2007). Pratylenchus penetrans is one of the most important nematodes of the Pratylenchus genus and attacks nearly 400 plant species, including high-value crops, such as grasses, forage crops, and fruit trees (Castillo and Vovlas 2007). All stages of P. penetrans, except eggs and first-stage juveniles, are vermiform and motile (Zunke 1990), and several generations of nematodes can develop during the lifespan of a crop.

Successful infection of RLNs depends on the secretion of a repertoire of proteins with diverse parasitism-related functions (Vieira et al. 2015, 2018). These proteins, known as effectors, are mainly synthesized in the nematode esophageal glands, and they are critical components of parasitism. The molecular mechanisms by which the effector-like proteins of the RLNs contribute to the parasitic behavior of the nematodes and their host targets or recognition receptors are largely unknown, although some putative biological roles have been suggested (Vieira et al. 2015, 2018).

†Corresponding author: L. G. Nemchinov; lev.nemchinov@usda.gov

Funding: This project was supported by U.S. Department of AgricultureAgricultural Research Service grant CRIS \#8042-21000-300-00D.

*The $e$-Xtra logo stands for "electronic extra" and indicates that supplementary materials are published online.

The author(s) declare no conflict of interest.

(C) 2020 The American Phytopathological Society
A set of endogenous cell wall-degrading enzymes that are often implicated in the softening and degradation of various components of the plant cell wall has been identified in most PPNs (Danchin et al. 2010). These include $\beta$-1,4-endoglucanases, pectate lyases, arabinogalactan endo-1,4- $\beta$-galactosidases, xylanases, and expansin-like genes (Danchin et al. 2010; Smant et al. 1998; Vieira et al. 2015). A few other genes or gene families, frequently recognized as part of the nematode-host secretome, have also been delineated by in silico analyses of P. penetrans (Vieira et al. 2015), including fatty acid and retinol-binding proteins, transthyretin-like proteins, venom allergen-like proteins, and a set of new candidate effector genes (Vieira et al. 2018). Nevertheless, despite the growing list of candidate effector genes of RLNs that has been compiled (Burke et al. 2015; Denver et al. 2016; Fosu-Nyarko and Jones 2016; Haegeman et al. 2011; Nicol et al. 2012; Vieira et al. 2015 , 2018), only a limited number of these genes have been experimentally validated or characterized.

Expansins, a family of nonenzymatic proteins, have been suggested to operate by loosening noncovalent interactions between components of the plant cell wall, making the individual components vulnerable to enzymatic attack (Cosgrove 2000). Expansins are typically plant proteins (Cosgrove 2000) and consist of an expansin domain fused to a C-terminal carbohydrate-binding domain. In nematodes, the first functional expansin (Gr-EXPB1) was reported in the cyst nematode Globodera rostochiensis (Kudla et al. 2005; Qin et al. 2004). When Gr-EXPB1 was expressed in transgenic tobacco, protein samples showed significant expansin activity, highlighting the potential involvement of $G r-E X P B 1$ in nematode parasitism. Subsequently, expansin-like proteins (EXPNs) have been reported in PPNs belonging to different clades (Danchin et al. 2010; Kikuchi et al. 2011; Opperman et al. 2008; Peng et al. 2013). The number of expansin-like genes identified in PPNs thus far varies. For example, in the root-knot nematode Meloidogyne incognita, a multigenic family of at least 20 different genes encoding expansins was identified (Danchin et al. 2010), whereas in other species, $<20$ expansins have been reported (Eves-van 
den Akker et al. 2016; Kikuchi et al. 2011; Opperman et al. 2008). Remarkably, expansin-like genes of the sedentary cyst nematodes G. rostochiensis (GrEXPB2) and Heterodera avenae (HaEXPB2 and $H a E X P B 1$ ) have been reported to have other functions in addition to modifying and softening the cell wall (Ali et al. 2015; Liu et al. 2016). The overexpression of GrEXPB2 induced chlorosis in Nicotiana benthamiana and necrosis in tomato and potato (Ali et al. 2015), whereas HaEXPB2 and HaEXPB1 triggered cell death in $N$. benthamiana (Liu et al. 2016). Furthermore, the EXPN of G. rostochiensis (GrEXPB2) was also able to suppress defense-related cell death in $N$. benthamiana and Nicotiana tabacum and inhibit resistance pathways mediated by the $\mathrm{N}$ and $\mathrm{Rx}$ nucleotide-binding site leucine-rich repeat (NB-LRR) proteins in $N$. benthamiana (Ali et al. 2015).

Previously, several transcripts encoding expansin-like genes were identified in the transcriptome of $P$. penetrans (Vieira et al. 2015). Transcripts encoding two EXPNs were later found to be localized within the esophageal glands of the nematode (Vieira et al. 2018), reinforcing their potential involvement in nematode parasitism. Nevertheless, a detailed characterization of the EXPNs in $P$. penetrans and a functional analysis of the members of this family are still lacking. Here, we showed that $P$. penetrans has a small multigene family encoding proteins with conserved expansinlike domains. We also applied a transient expression methodology to characterize the putative biological roles of one of the genes in this family, $P p-E X P B 1$, that was highly expressed during the interaction of nematodes with different host plants. The methodology relied on using the potato virus X (PVX)-based vector, a well-established tool for high-throughput functional screening and rapid in planta studies (Lacomme and Chapman 2008), and $N$. benthamiana, a model plant for host-pathogen interactions (Goodin et al. 2008). Phenotypically distinct plants expressing effector proteins were further investigated by next generation sequencing to reveal the changes in their transcriptome profiles triggered by the effector protein. Our experiments demonstrated that Pp-EXPB1 substantially modulated the host defensive response, thus indicating a key role of the effector in both nematode parasitism and the effective recognition of Pp-EXPB1 by the plant immune system.

\section{MATERIALS AND METHODS}

Nematode isolate. P. penetrans isolate NL 10p RH, collected in Beltsville, Maryland, U.S.A., was used for all experiments. This isolate was maintained and multiplied in vitro in the roots of corn (Zea mays L. 'Iochief') growing in Murashige and Skoog basal medium containing vitamins and 3\% (wt/vol) sucrose ( $\mathrm{pH} 5.8)$ that was solidified with $1.5 \%$ (wt/vol) agar (Sigma-Aldrich). Nematodes were extracted from infected corn roots as described by Vieira et al. (2015).

Identification and cloning of the $P$. penetrans expansinlike genes. $P$. penetrans transcripts encoding expansin-like genes were identified using the transcriptome assembly previously generated for the isolate NL 10p RH (Vieira et al. 2015). Primers were then designed to clone both genomic DNA and complementary DNA (cDNA) sequences of the corresponding predicted transcript sequences (Supplementary Table S1). DNA extraction was performed from mixed stages (eggs, juveniles, females, and males) of $P$. penetrans using the PureLink Genomic DNA Mini kit (Invitrogen) following the manufacturer's instructions. Total RNA was extracted from mixed life stages of $P$. penetrans using the RNeasy Plant Mini kit (Qiagen) according to the manufacturer's instructions. RNA was then treated with RNase-Free DNase before reverse transcription (RT). First-strand cDNA was synthesized using the iScript first-strand synthesis kit (Bio-Rad) following the manufacturer's instructions. Each corresponding PCR product was ligated into the TOPO TA Cloning kit (Invitrogen), and positive clones were sequenced in both directions and verified by automated
Sanger sequencing at the Macrogen USA facility in Maryland. All genomic clone sequences were deposited in GenBank at the NCBI under the accession numbers MN531558 to MN531561.

Sequence analysis. cDNA and genomic sequences were aligned using MUSCLE (Edgar 2004), and gene schematics were generated with the Exon-Intron Graphic Maker available at WormWeb.org. The nucleotide and translated amino acid sequences were analyzed for similarity to other genes and proteins using BLAST analyses against the NCBI nonredundant nucleotide and protein databases (https://www.ncbi.nlm.nih.gov/). Protein sequence analyses were conducted using the following programs: SignalP 4.0 for the prediction of protein signal peptides (Petersen et al. 2011) and CLC Main Workbench v. 8.0 software for the determination of the protein molecular mass and theoretical isoelectric points. InterPro scan was applied using the publicly available software package (https://www.ebi.ac.uk/interpro/). The phylogenetic relationships were estimated using maximum likelihood (ML) analysis. The best model for protein evolution, which was determined with AMINOSAN (Tanabe 2011), was the "Whelan and Goldman" model with discrete $\gamma$-distribution (number of $\gamma$ categories $=8$ ). The robustness of ML analysis was inferred using 1,000 bootstrap replicates.

Differential expression of the $P$. penetrans expansin-like genes. Transcriptome data of $P$. penetrans-infected plants (Vieira et al. 2015, 2019) were extracted from the NCBI Sequence Read Archive under the BioProject ID PRJNA304159 and BioProject ID PRJNA547347. The expression of the different expansin-like genes was retrieved as reads per kilobase of transcript per million mapped reads values and presented as the average value \pm standard error. Semiquantitative RT-PCR analyses were performed using total RNA extracted from nematode-infected roots of alfalfa and soybean hairy roots at different time points following the same methodology as described by Vicente et al. (2019). The following genes were used as references: Ubiquitin-3 for soybean hairy roots, NP_001237047 for alfalfa, and $18 S$ for $P$. penetrans (Supplementary Table S1).

Generation of recombinant $P V X$ constructs and their expression in planta. To express $P p-E X P B 1$ in planta, EcoRVflanked PCR products were initially cloned into the pCRII-TOPO vector (ThermoFisher Scientific) according to the manufacturer's directions and verified by automated Sanger sequencing at the Macrogen USA facility. Then, the vector was digested with the EcoRV restriction enzyme, gel purified, and subcloned into the EcoRVlinearized PVX-based vector pP2C2S (obtained from D. Baulcombe, Sainsbury Laboratories, Norwich, England). The integrity of all PVX clones was verified by automated sequencing. pP2C2S plasmids were linearized with SpeI, and capped transcripts were generated from the cDNA clones using the Ambion T7 mMessage Machine kit (ThermoFisher Scientific). PVX/Pp-EXPB1 transcripts were mechanically inoculated into fully expanded leaves of three $N$. benthamiana plants. Transcripts were also produced from $\mathrm{pP} 2 \mathrm{C} 2 \mathrm{~S}$ plasmids without inserts ("empty" PVX vector) and inoculated into three plants to serve as controls representing a wild-type (WT) PVX infection (PVX-WT). Three more plants were inoculated with buffer as negative ("healthy") controls. Inoculated plants were monitored daily for symptoms. The inoculation experiments that included all sets of plants of each variant were repeated three times. At 14 days after inoculation (DAI), the leaves were photographed, collected, snap frozen in liquid nitrogen, and stored at $-80^{\circ} \mathrm{C}$ until RNA extraction.

Agrobacterium-mediated transient expression. The binary pGD vector for the transformation of Agrobacterium tumefaciens strain EHA105 was supplied by Rosemarie Hammond (originally acquired from Michael Goodin, University of Kentucky, Lexington, $\mathrm{KY}$ ). The $P$ p-EXPB1 coding sequence was amplified with PCR primers flanked with HindIII and BamHI restriction sites and cloned into the pCRII-TOPO vector. Plasmids were digested with the same restriction enzymes, gel purified, and subcloned into the HindIII/ BamHI-linearized pGD vector. At each step, the integrity of cloning was verified by automated Sanger sequencing. The transformation 
of A. tumefaciens and agroinfiltration procedures were performed as described by Goodin et al. (2008).

Protein identification by immunoassay. Western blot assays were performed with the original tissues infected with $\mathrm{PVX} / \mathrm{Pp}$ EXPB1 essentially as described in Nemchinov and Natilla (2007). Membranes were probed with the affinity-purified polyclonal antibodies (Abs) developed against synthetic peptide derived from the sequence of $P p$-EXPB1 (TASKTLTVPINNKC). The peptide antigen and Abs were produced by GenScript according to the company protocols (GenScript Inc.). Reactions were developed with NBT/BCIP (5-Bromo-4-chloro-3-indolyl phosphate/nitro blue tetrazolium) phosphatase substrate (Thermo Fisher Scientific, U.S.A.).

Trypan blue staining assay. The occurrence of cell death in $N$. benthamiana leaves was examined using trypan blue staining of PVX and PVX/Pp-EXPB1-infected leaves that were harvested 14 DAI. Leaves were stained according to the protocol described in Koch and Slusarenko (1990).

RNA-sequencing experiments. For Illumina RNA-sequencing (RNA-seq) experiments, total RNA extraction was performed with the RNeasy Plant Mini kit (Qiagen) following the manufacturer's instructions. Three biological replicates (five pooled leaves from each of the three plants represented one replicate) were used for the experiment. The purity and quantity of the samples were verified with a NanoDrop spectrophotometer (ThermoFisher Scientific). RNA-seq was performed by Novogen USA. cDNA libraries were generated using a poly (A) selection method, and paired-end reads $(2 \times 150 \mathrm{bp})$ were obtained on the Illumina HiSeq 2500 platform.

Read mapping and functional analysis. The paired-end reads were mapped onto the available genome sequence of $N$. benthamiana downloaded from the Sole Genomic Network (https://solgenomics.net/organism/Nicotiana_benthamiana/genome) (Bombarely et al. 2012) using the RNA-seq analysis module in CLC Genomic Workbench 8.0.2 software (Qiagene). Gene annotations were based on BLASTX hits with the Swiss-Prot database. The DESeq2 package from Bioconductor in the $\mathrm{R}$ statistics suite $(\mathrm{R}$ version 3.4.0) was used to estimate the sample quality and expression levels of the genes. Genes with a fold change of $>2$ and a false discovery rate (FDR) of $<0.05$ were counted as differentially expressed.

Part of the computational analysis (Kyoto Encyclopedia of Genes and Genomes [KEGG] and gene ontology [GO] enrichment) was performed by the RNA-seq provider Novogene. Significant GO enrichment terms were detected by Fisher's exact test (FDR $<0.05$ ).

Verification of transcriptome data by quantitative realtime PCR. Quantitative PCR (qPCR) was performed with arbitrarily selected genes to confirm the transcriptomic data. Primers (Supplementary Table S1) were designed using the online Realtime PCR tool (Integrated DNA Technologies Inc.; https://www.idtdna. com/scitools/Applications/RealTimePCR/). cDNA for qPCR analyses was made using the SuperScript III First-Strand Synthesis System with oligo d(T) (ThermoFisher Scientific) and the same RNA samples that were used for RNA-seq. Amplification was conducted with a CFX96 real-time system machine (Bio-Rad) with three biological replicates using the following parameters: $95^{\circ} \mathrm{C}$ for $10 \mathrm{~min}(1 \mathrm{cycle})$ followed by $95^{\circ} \mathrm{C}$ for $10 \mathrm{~s}$ and $60^{\circ} \mathrm{C}$ for $45 \mathrm{~s}(40$ cycles). The $\Delta \Delta \mathrm{C}(\mathrm{T})$ method $\left(2^{\left.-\Delta \Delta C_{\mathrm{T}}\right)}\right.$ was used for the analysis of relative expression (Livak and Schmittgen 2001). The L23 and $P P 2 A$ genes (Liu et al. 2012) from $N$. benthamiana were used as internal reference genes to determine relative expression values.

\section{RESULTS}

Identification of the expansin-like genes in $P$. penetrans. Previous analyses of the transcriptome of $P$. penetrans (Vieira et al. 2015) led to the identification of several transcripts encoding EXPNs. To resolve the number of genes in this family in $P$. penetrans, detailed in silico analyses were conducted, which revealed the presence of at least four members in this family. The predicted transcript sequences included open reading frames ranging from 543 to $987 \mathrm{nt}$, with the remaining transcript features summarized in Table 1 . To confirm their eukaryotic origin, the genomic clones of these transcripts were sequenced, and BLAST analyses were carried out against the available skimming genome of $P$. penetrans (Denver et al. 2016). For each transcript, the corresponding genomic sequence was sequenced (Fig. 1A), revealing the presence of two to five introns and thus, excluding potential contamination from prokaryotic origins.

The predicted proteins of this small gene family ranged from 180 to 328 amino acids, with molecular weights varying between 18.7 and $32.9 \mathrm{kDa}$ (Table 1). All of the deduced proteins displayed an $\mathrm{N}$ terminal signal peptide for secretion, suggesting that the four proteins are likely secreted by the nematode. InterPro scan analyses showed that all four mature predicted proteins contained a $\beta$-expansin domain (DPBB_1; IPR009009) in the C-terminal region, whereas three of the four proteins also had a carbohydrate-binding type-2 domain (CBM2; IPR001919) at the N-terminal region (Fig. 1B). The fulllength proteins showed 35.17 to $62.5 \%$ identity among each other, whereas their expansin domains shared 69.3 to $77.8 \%$ identity (Supplementary Fig. S1). Sequence alignment of the $P$. penetrans expansin domains with expansin and expansin-like domains of other PPNs showed the presence of several conserved cysteines among different species of nematodes (Fig. 1C). Several conserved signatures of the expansin family in plants, such as HFD and T(F/W)YG domains, were also found (Cosgrove 2000). The four expansin-like genes of $P$. penetrans identified in this study were named $P p-E X P B-1$ to $P p-E X P B-4$ and deposited at GenBank (NCBI) with the accession numbers of MN531558 to MN531561, respectively.

BLAST searches of the expansin domain of Pp-EXPB1 against the nonredundant protein dataset at the NCBI showed that the $P$. penetrans sequence was most similar to expansin and expansinlike sequences from several cyst nematodes (up to $58.5 \%$ similarity) (Supplementary Table S2). When BLAST searches were performed against other organisms, the closest BLAST hit corresponded to the hypothetical proteins from different bacteria of the order Actinomycetales, phylum Actinobacteria (37.6 to $33.9 \%$ similarity) (Supplementary Table S2); BLAST searches against fungi or plants revealed a very low level of identity (Supplementary Table S2).

To understand the phylogenetic relationship between $P$. penetrans EXPNs and EXPNs from other nematodes, bacteria, fungi, and plants, an ML analysis was performed (Fig. 2). All $P$. penetrans EXPNs were clustered in the same monophyletic group with the EXPNs derived from other PPNs and clearly separated from the other taxa.

Differential expression of $P$. penetrans expansin-like genes. To evaluate the relative expression of the four EXPN genes, we retrieved available transcriptomic libraries of $P$. penetrans (Vieira et al. 2015) and different nematode-infected plant species (Vieira et al. 2015, 2019). Transcript abundance was confirmed for all four of the members, with $P p-E X P B 1$ the most highly expressed compared with the other genes followed by $P p-E X P B 2$ (Fig. 3A to C). Transcripts of both $P p-E X P B 1$ and $P p-E X P B 2$ were previously localized within the esophageal glands of $P$. penetrans (Vieira et al. 2018). To substantiate our in silico analyses, semiquantitative RTPCR was performed with independent cDNA libraries obtained from mixed stages of $P$. penetrans from alfalfa and soybean hairy roots infected with nematodes at different time points. The expression pattern of $P p-E X P B 1$ and the other gene members was consistent with the RNA-seq results (Fig. 3D to F).

Transient expression of the $P p-E X P B 1$ candidate effector gene in $N$. benthamiana plants via the PVX-based vector. The overexpression of nematode effectors can trigger atypical phenotypes in plants, which may correspond to the activity of effectors during nematode parasitism (Chen et al. 2018). Affected plants may exhibit cell death phenotypes, chlorosis, necrosis, tissue browning, stunting, and other gross morphological 
TABLE 1. Summary of the expansin-like genes of Pratylenchus penetrans, including their genomic and coding region features ${ }^{\mathrm{a}}$

\begin{tabular}{|c|c|c|c|c|c|c|c|c|c|c|c|c|}
\hline Expansin like & Transcript length & $5^{\prime}$ UTR & CDS (nt) & $3^{\prime}$ UTR & Poly-A & Genome (nt) & Protein (aa) & SP & PI & MW (kDa) & CBM2 domain & DPBB domain \\
\hline Pp-EXPB1 & 723 & 43 & 543 & 118 & Yes & 808 & 180 & Yes & 9.08 & 18.74 & - & $56-173$ \\
\hline Pp-EXPB2 & 1,170 & 38 & 972 & 150 & Yes & 1,170 & 323 & Yes & 8.58 & 32.32 & $25-118$ & $202-316$ \\
\hline Pp-EXPB3 & 1,327 & 30 & 987 & 291 & Yes & 1,146 & 328 & Yes & 5.42 & 32.95 & $25-121$ & $232-320$ \\
\hline Pp-EXPB4 & 1,117 & 21 & 936 & 150 & Yes & 1,140 & 311 & Yes & 8.94 & 30.62 & $22-115$ & $190-301$ \\
\hline
\end{tabular}

${ }^{a}$ UTR, untranslated region; CDS, coding sequence; SP, signal peptide; PI, isoelectric point; MW, molecular weight; and -, no CBM2 domain present.
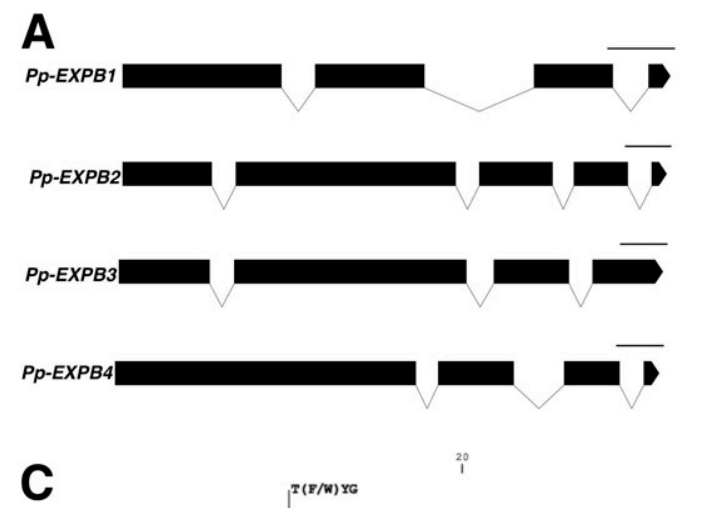
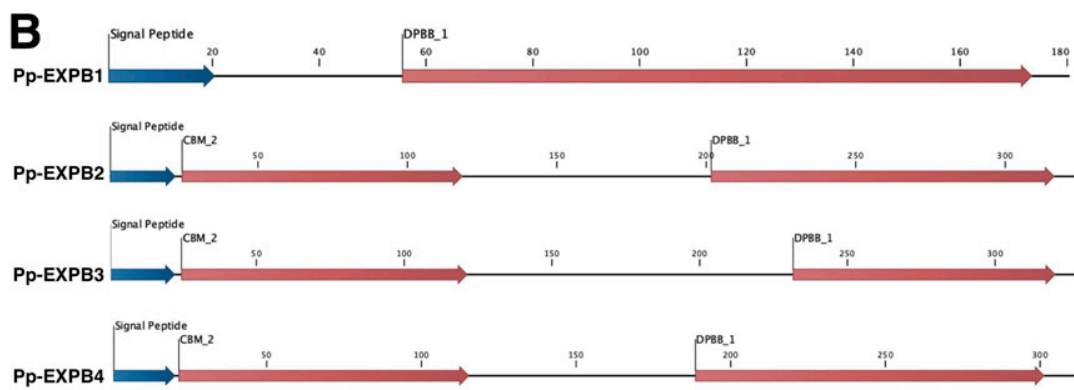

$i^{40} \quad 1000$

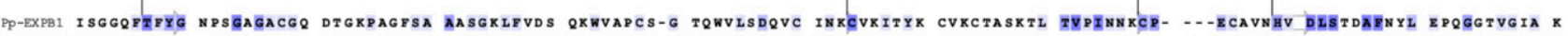

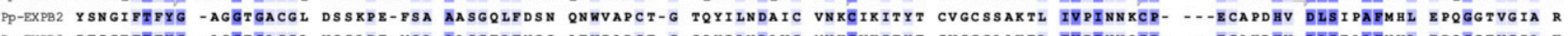

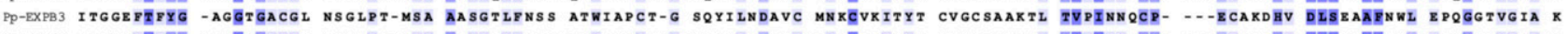

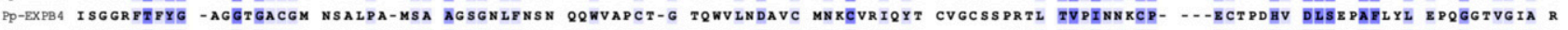
Globodera rostochiensis FTNGVFTFE -ATGRSACGL DAGKPK-MSA SVSGKLFKSD GQWKNACRID QQYMLDDPIC KNICVRIDYK -

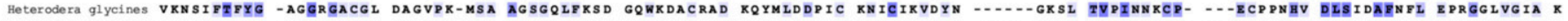

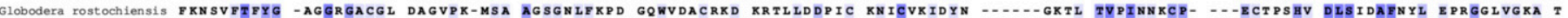
Nicotiana tabacum FLPAVATWYG DPDGAGSD-- -

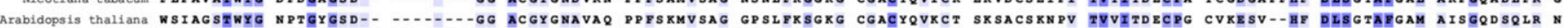

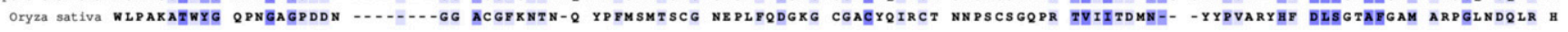

Fig. 1. Molecular characterization of the expansin-like genes of Pratylenchus penetrans. A, Gene structure representation of the different expansin-like genes of $P$. penetrans. Exons are represented with dark boxes; introns are represented with lines, and their relative positions and respective sizes are also indicated. B, Protein structures of the different expansin-like proteins of P. penetrans showing the position of the signal peptides, the carbohydrate-binding type-2 domain (CBM_2), and the expansin domain (DPBB_1). C, Multiple sequence alignment of P. penetrans expansin-like domain sequences (Pp-EXPB1 to Pp-EXPB4) with the expansin and expansin-like protein domains from other plant-parasitic nematodes (Globodera rostochiensis: CAC83611 CAC84564; and Heterodera glycines: ADL29728) and plants (Nicotiana tabacum: AF333386; Arabidopsis thaliana: AC001229; and Oryza sativa: AAB61710). Conserved cysteine (C) and key signatures of the expansin family of plants are marked at the top of the alignment. Conserved residues among species are indicated with dark blue shading and dots, whereas similar residues are represented in light blue using a $60 \%$ similarity threshold for shading.

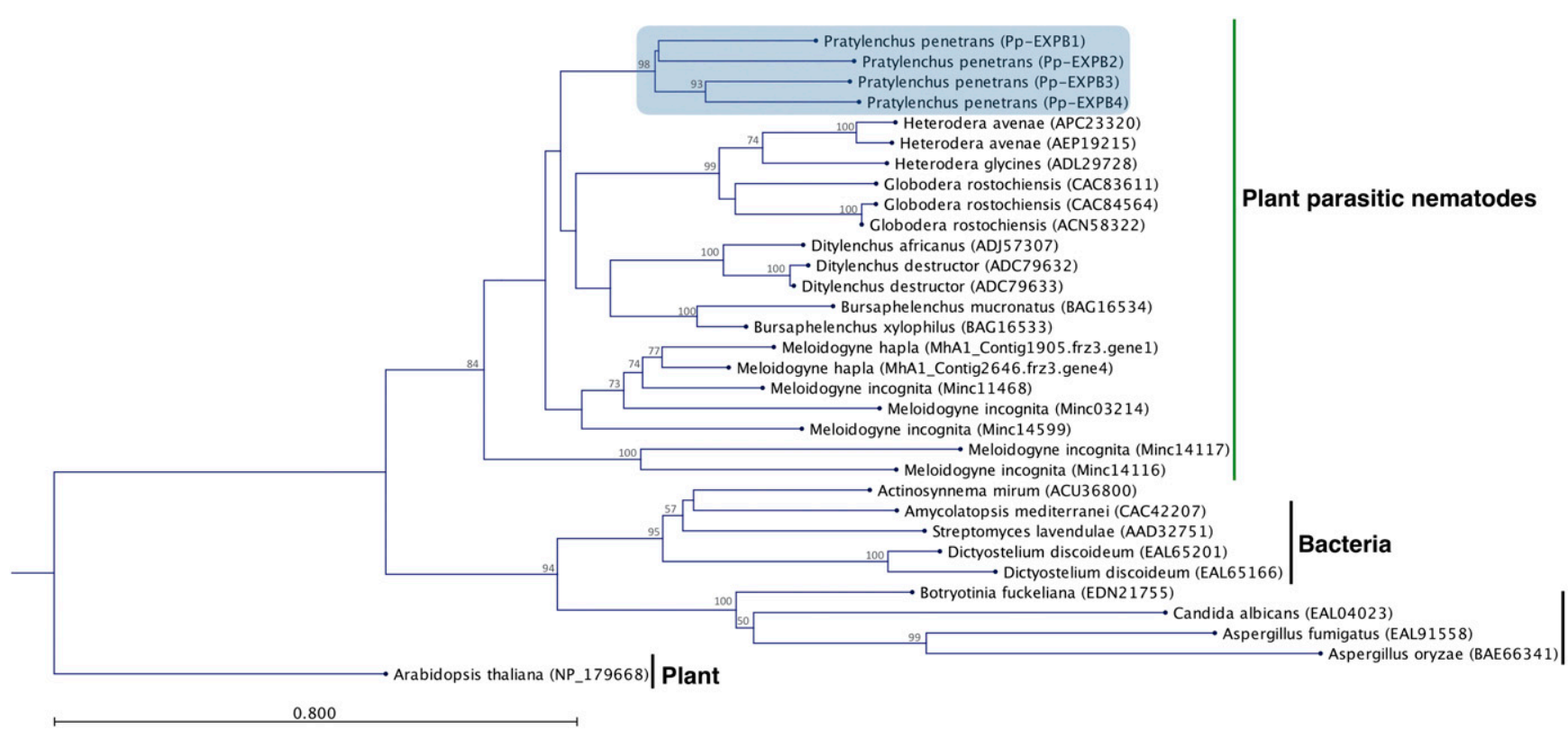

Fig. 2. Phylogenetic relationship of the expansin-like proteins of Pratylenchus penetrans to expansin and expansin-like proteins found in other species. All protein sequences were obtained from the NCBI with the exception of the sequences for Meloidogyne incognita and Meloidogyne hapla, which were obtained from their respective genome sequencing datasets. The phylogenetic tree was generated by maximum likelihood analyses with 1,000 bootstrap replicates. 
changes. Therefore, because the expression of $P p$-EXPBl was consistently higher than that of the other members of this family, this gene was chosen for further functional analyses. The full-length coding sequence (CDS) of $P p-E X P B 1$, including its signal peptide cleavage site, was amplified and cloned into the PVX-based virus vector system for plant expression. P. penetrans $P$ p-EXPB1 was then tested for its ability to elicit a plant response using a PVX-based transient expression assay in $N$. benthamiana.

At 14 DAI, $N$. benthamiana plants expressing $P p-E X P B 1$ with the native signal peptide were notably dwarfed (Fig. 4B) and displayed chlorosis and multiple leaf abnormalities (Fig. 4D), including small localized necrotic spots that resembled a hypersensitive response (HR). The stems of the plants infected with the recombinant PVX vector displayed visible lesions (Fig. 4F); however, these lesions were absent from the stems of the PVX-WT-infected plants (Fig. $4 \mathrm{E})$. Later in the course of infection, $\mathrm{PVX} / \mathrm{Pp}-\mathrm{EXPB} 1$ plants developed malformed, abortive flowers (Fig. 4H); their root systems were shortened and contained dark areas of necrotic tissue (Fig. 4J). Plants infected with the PVX vector without the insert displayed a typical mosaic-like phenotype characteristic of the WT PVX infection (Fig. 4A and C).

To ensure that the leaves of the PVX/Pp-EXPB1-infected plants exhibited HR-like defense reactions, we stained them with trypan blue to visualize the induction of cell death. Multiple micro-HR lesions disseminated across the entire leaf surface were observed only in the leaves of the PVX/Pp-EXPB1-infected plants (Fig. 5B and D).

The expression of $P p$-EXPBI in PVX-infected plants was confirmed by semiquantitative RT-PCR using RNA extracted from systemically infected noninoculated leaves (Fig. 6A). This experiment indicated that the induction of the corresponding phenotype was directly related to the replication of the recombinant PVX virus. To validate that the PVX-expressed $P p-E X P B 1$ gene was processed into the protein, we performed western blot analysis with Abs specific to the internal epitope of Pp-EXPB1. The assay demonstrated that the effector protein was successfully produced in $N$. benthamiana plants (Fig. 6B), therefore verifying that the observed phenotypic abnormalities and transcriptomic changes described below were attributed to the PVX-expressed Pp-EXPB1 protein.

Expression of the $P p-E X P B 1$ candidate effector gene in $N$. benthamiana leaves through an Agrobacterium binary vector. To further investigate and confirm the HR-like symptomatology observed in the $P p-E X P B 1$-expressing plants, the gene was cloned into the binary vector pGD (Goodin et al. 2008) and used for A. tumefaciens-mediated transient expression assays in $N$. benthamiana. The agroinoculation of plants with the $\mathrm{pGD} / P p$ EXPB1 construct caused characteristic HR-like cell death symptoms on the infiltrated leaves of $N$. benthamiana 7 DAI (Fig. 7A), thus indicating that the Pp-EXPB1 candidate effector was potentially recognized by a resistance protein present in the $N$. benthamiana plants and capable of eliciting an HR. Because no HR was observed on the leaves infiltrated with the empty pGD vector (Fig. 7B), the experiment also confirmed that the observed plant response was not dependent on the presence of the genus Agrobacterium. The presence of the $P p-E X P B 1$ transcripts in the leaves was confirmed by qPCR (Fig. 7C).

RNA-seq and transcriptome profiling of $N$. benthamiana plants infected with the recombinant $P V X / P p-E X P B 1$ vector. The induction of cell death by plant pathogens is often

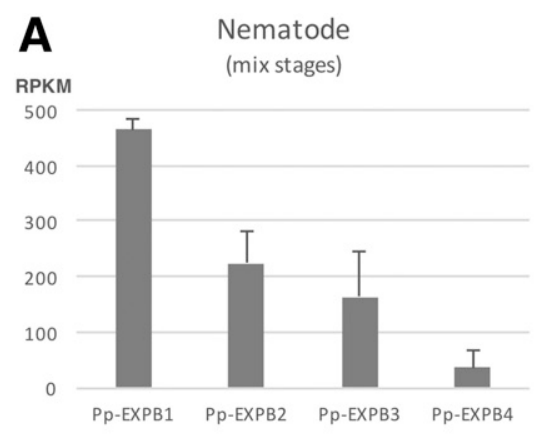

D

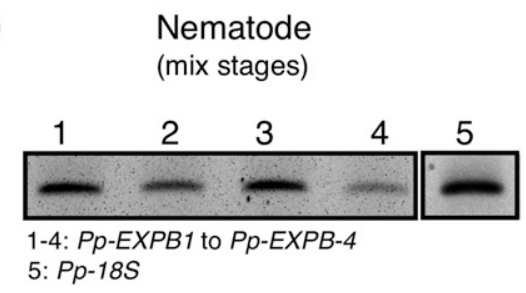

B

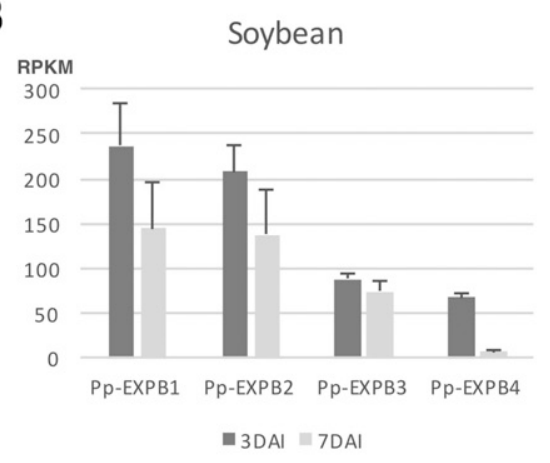

$\mathbf{E}$

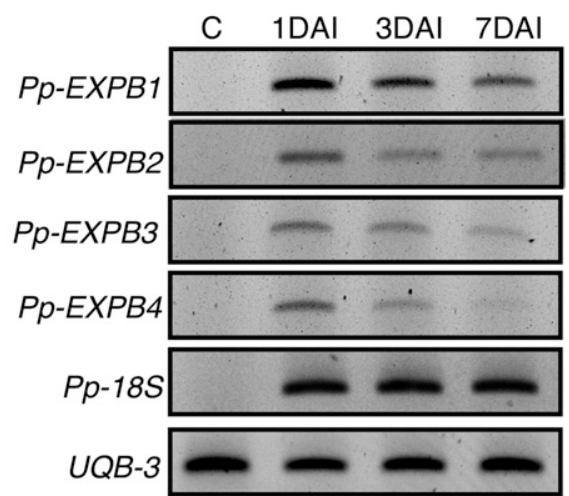

C

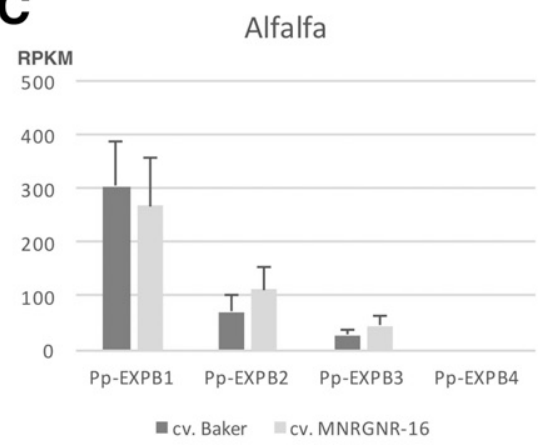

$\mathbf{F}$

Alfalfa

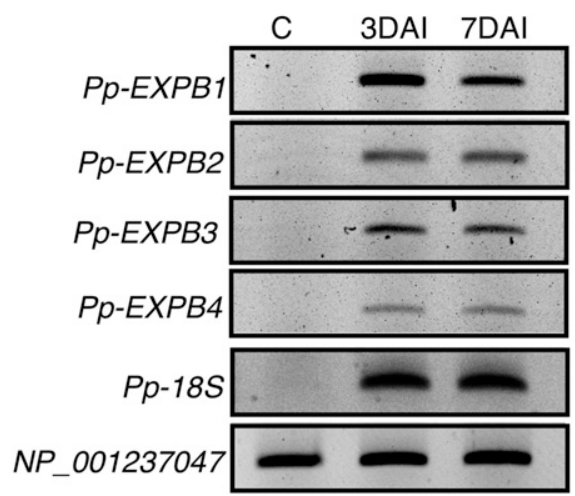

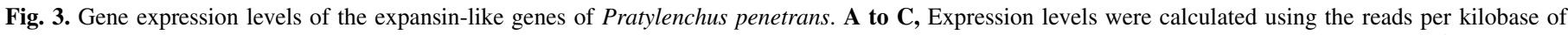

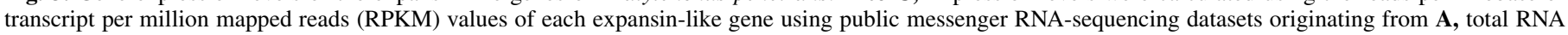

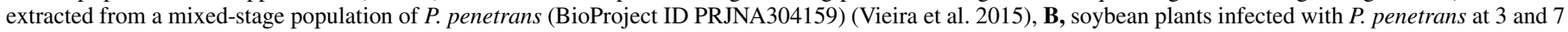

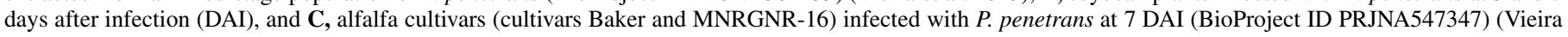

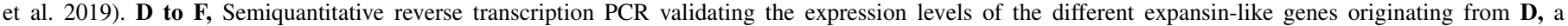
population of mixed-stage nematodes; E, soybean hairy roots collected at 1, 3, and 7 DAI; and F, alfalfa roots (Baker) collected at 3 and 7 DAI. 


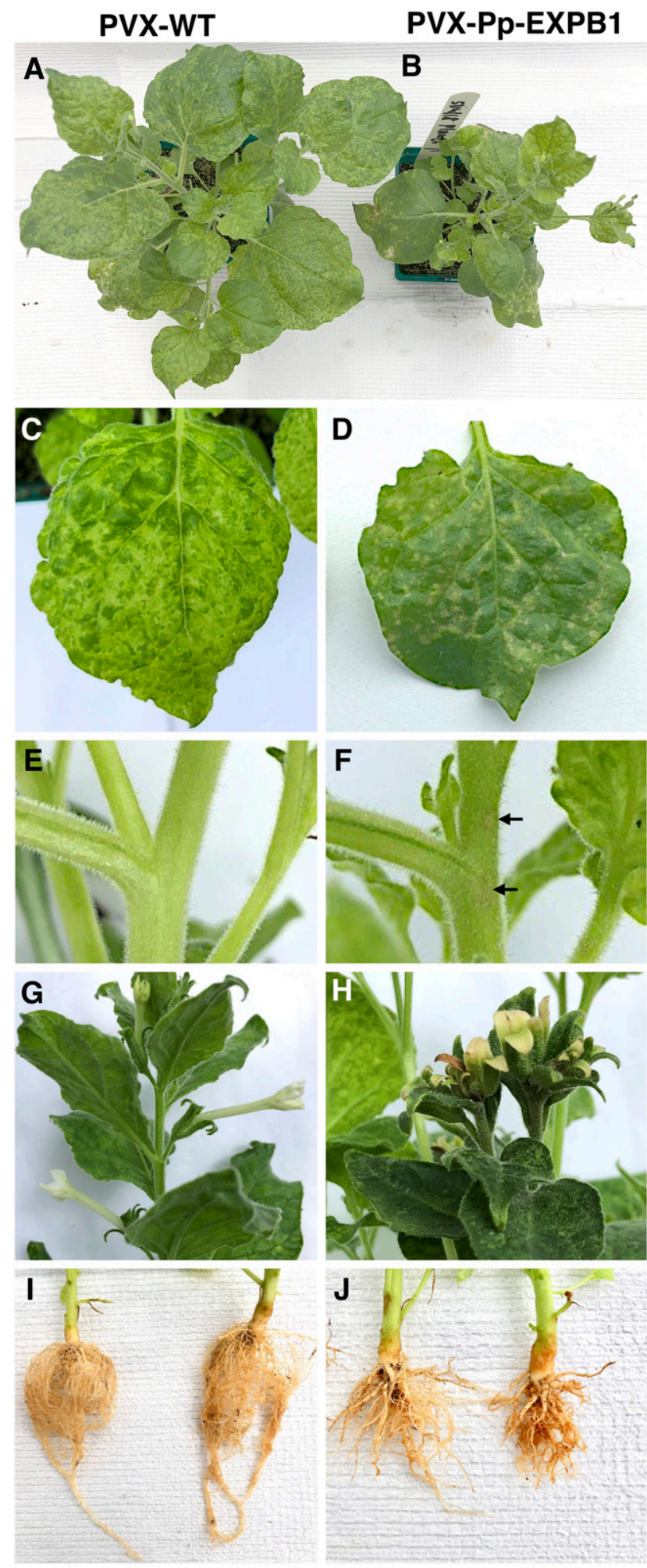

Fig. 4. Phenotypic changes in Nicotiana benthamiana plants infected with the recombinant PVX/Pp-EXPB1 virus. B, Expression of PVX/Pp-EXPB1 induced dwarfing in $N$. benthamiana plants, which was absent in A, plants infected with PVX-WT. C, Leaves expressing PVX/Pp-EXPB1 displayed necrotic spots that were not observed in D, PVX-WT plants. E and F, Lesionlike symptoms observed on the stems of $N$. benthamiana plants infected with PVX/Pp-EXPB1 transcripts (indicated by arrows in F). E, Stems of N. benthamiana plants infected with transcripts generated from the empty PVX vector did not display any lesion-like symptoms. H, Plants expressing PVX/ Pp-EXPB1 had malformed and misshapen leaves compared with the normally developed leaves in G, plants expressing PVX-WT. J, Roots expressing PVXPpEXPB1 were reduced in size and contained dark areas of necrotic tissue unlike I, roots of the PVX-WT plants. Photographs A to F were taken 14 days after inoculation; photographs $\mathbf{G}$ to $\mathbf{J}$ were taken 45 days after inoculation. the outcome of the recognition of such pathogens by the plant immune system, which involves a variety of receptors and signal transduction pathways (Jones and Dangl 2006). To assess the overall molecular mechanisms behind the $P p$-EXPB1-induced phenotypic changes, we performed differential gene expression analysis of the transcripts produced by Illumina RNA-seq. Plants inoculated with PVX-WT were used as a control.

Metrics of RNA-seq data. A total of 286 million reads were generated from six messenger RNA-seq libraries. The reads were mapped to the representative genome of PVX (NCBI accession number M72416.1) and publicly available genome of N. benthamiana (https://solgenomics.net/organism/Nicotiana_benthamiana/genome). The average percentage of the total number of reads mapped to each respective genome is shown in Supplementary Table S3.

Identification of differentially expressed transcripts and their functional characterization. A number of differentially expressed genes (DEGs) were identified in the PVX/Pp-EXPBIinfected plants (Fig. 8A and Supplementary Table S4). DEGs unique to the PVX/Pp-EXPBI plants were depicted against the background of a WT PVX infection. The total number of DEGs and their broad biological roles demonstrated the wide-ranging influences of the plant-produced nematode EXPN on the gene expression patterns of $N$. benthamiana (Supplementary Table S4). A total of 3,411 DEGs were detected in the PVX/Pp-EXPB1 versus PVX-WT plants, including 1,350 genes that were significantly (fold change $>2$ ) up-regulated and 2,061 genes that were down-regulated (Fig. 8A). A larger number of down-regulated DEGs than upregulated DEGs indicated that the overall impact of $P p-E X P B 1$ lies in the extensive suppression of the host biological and cellular processes.

An in-depth analysis of the down-regulated DEGs in the PVX/Pp$E X P B 1$ plants showed that most of the enriched GO terms represented genes of chloroplast origin (Supplementary Table S5A). These included key genes involved in photosynthetic processes of electron transport, energy conversion, and carbon dioxide fixation

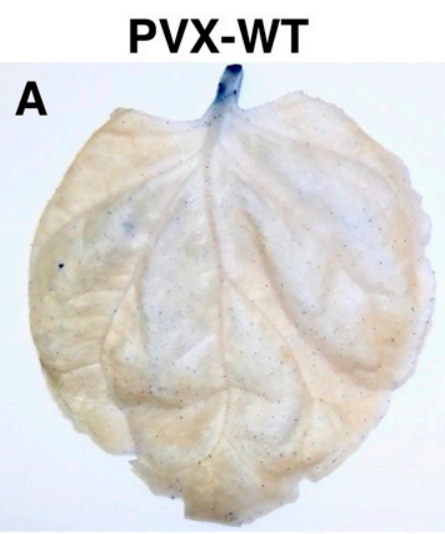

\section{PVX-Pp-EXPB1}
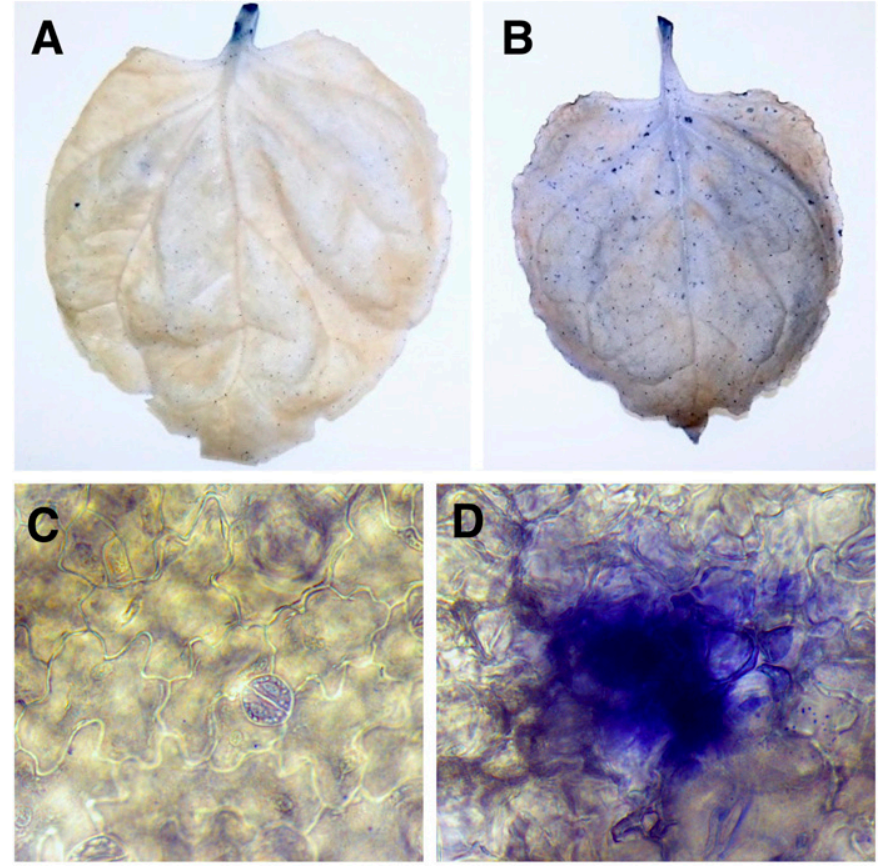

Fig. 5. Occurrence of cell death in leaves of the PVX/Pp-EXPB1-infected Nicotiana benthamiana plants. Cell death was visualized by trypan blue staining. B and D, PVX/Pp-EXPB1-infected plants. A and C, PVX-WT. C and $\mathbf{D}$ correspond to the enlarged images of leaf fragments PVX-WT and PVX/, respectively. Leaves were collected at 10 days after infection. 
(Supplementary Tables S4 and S5). In total, >43\% (884 DEGs) of all down-regulated DEGs represented genes of chloroplastic origin. Other down-regulated DEGs were outlined by the genes involved in a wide range of key biological processes, including transcription,
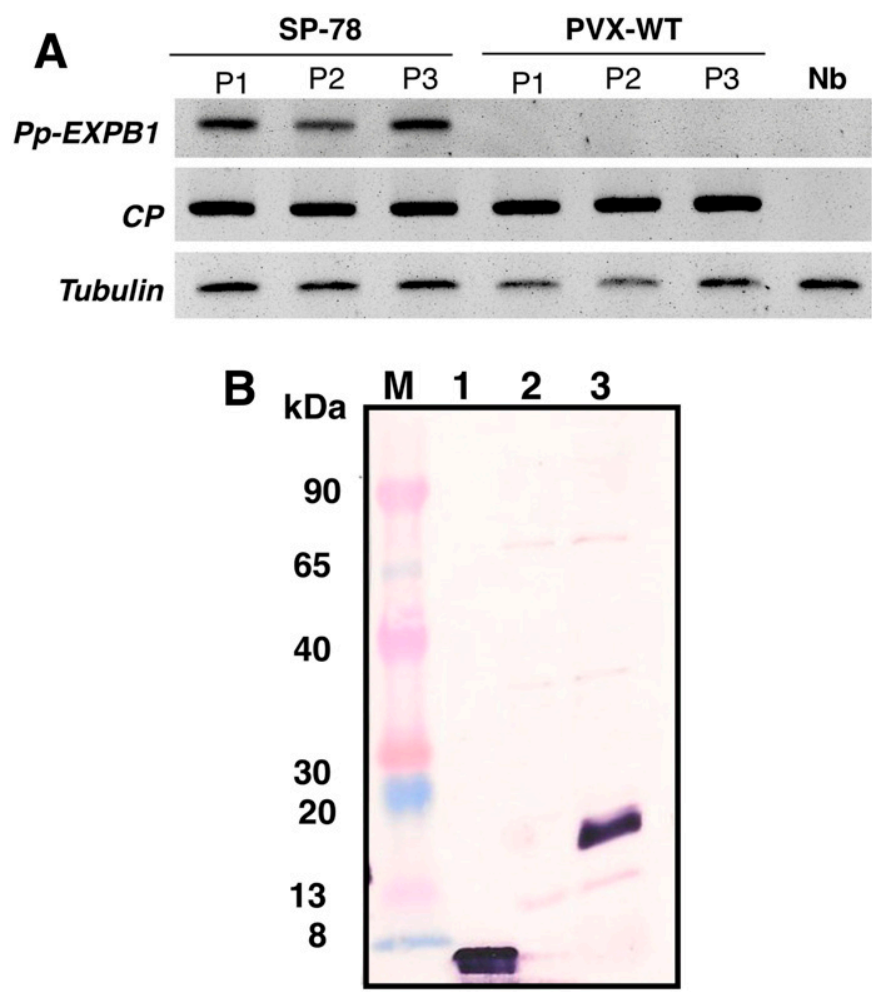

Fig. 6. Detection of PVX-WT and PVX/Pp-EXPB1 in the leaves of infected Nicotiana benthamiana plants. A, Detection of PVX-WT and PVX/Pp-EXPB1 transcripts by semiquantitative reverse transcription PCR. P1, P2, and P3 correspond to different leaves collected from three independently inoculated plants. $\mathrm{Nb}$ corresponds to a noninoculated $N$. benthamiana plant. Primers used for quantitative PCR were derived from the nucleotide sequence of the $P p$ EXPB1 gene (Pp-EXPB1) and sequence of the PVX coat protein $(\mathrm{CP})$. The plant tubulin 1 (Tubulin) was used as a reference. B, Protein accumulation was determined by western blot assay using antibodies specific to the internal epitope of the Pp-EXPB1 (Materials and Methods). M indicates the ColorBurst molecular weight marker (Sigma-Aldrich), 1 indicates the Pp-EXPB1 antigen, 2 indicates the $N$. benthamiana leaves inoculated with PVX-WT, and 3 indicates $N$. benthamiana leaves inoculated with PVX-Pp-EXPB1. translation, hormone regulation, membrane transport, and mitochondrial biogenesis (Supplementary Table S5A). The global downregulation of photosynthesis genes was proposed in 2010 to contribute to an adaptive response against biotic stresses by diverting main plant recourses toward immediate defense needs (Bilgin et al. 2010).

We, therefore, examined the transcriptional response of known defense-associated genes to identify genes and pathways that could potentially benefit from the down-regulation of photosynthetic processes as proposed by Bilgin et al. (2010). KEGG pathway analysis (Fig. 8B and C) showed that two categories among the upregulated genes were significantly enriched in the PVX/Pp-EXPB1 plants: protein processing in endoplasmic reticulum (ER) and plant-pathogen interaction (Fig. 8B and Supplementary Table S6A).

The major role of the ER in plant stress pathways has been well documented (Brandizzi et al. 2014; Kørner et al. 2015). The KEGG map of the protein processing in ER term included genes encoding proteins with critical roles in folding, sorting, and degrading proteins in the ER: calreticulin, which binds to misfolded proteins; heat shock proteins 70 and 90, which participate in the ERassociated degradation process; E3 ubiquitin-protein ligase, which is required for ubiquitination; a DnaJ homolog, which is needed for the proper folding, trafficking, or degradation of proteins; and others (Supplementary Table S6A).

The KEGG map of the plant-pathogen interaction term identified some key genes encoding proteins involved in signaling pathways of innate immunity and defense responses to the expression of Pp-EXPB1: LRR receptor-like serine/threonine-protein kinase, pathogenesis-related protein 1, WRKY transcription factor 33, receptor-like protein EIX2, RPM1-binding proteins interacting with the disease resistance gene product RPM1 (Boyes et al. 1998), calcium-binding proteins, and heat shock protein $90 \mathrm{kDa}$ (Supplementary Table S6A).

The categorization of all DEGs obtained in this study revealed a number of important defense-related genes that were up-regulated in PVX/Pp-EXPB1 (Supplementary Table S4). Among them were two highly up-regulated genes encoding the putative late blight resistance protein homologs R1B-19 (Niben101Scf03069g01016.1) and RB-17 (Niben101Scf01398g00006.1) (Supplementary Table S4), a homolog of the TMV resistance protein N (TMV-N; Niben101Scf08390g01002.1), and five genes encoding endochitinases, all of which are capable of conferring enhanced resistance to different plant pathogens. The up-regulation of the latter DEGs, especially those encoding orthologs of the late blight resistance

\section{pGD/Pp-EXPB1}

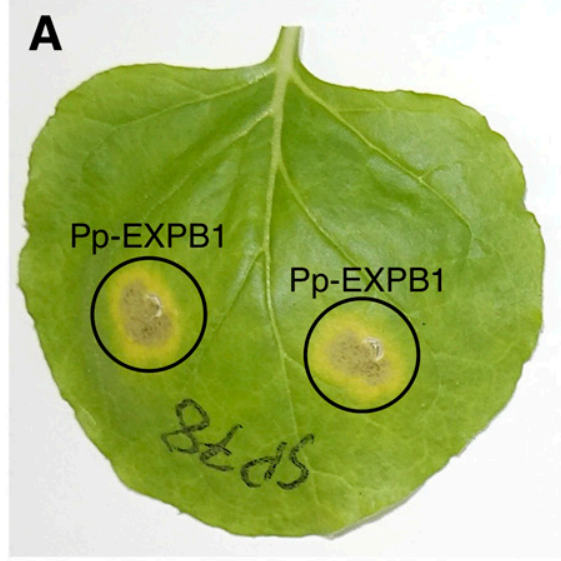

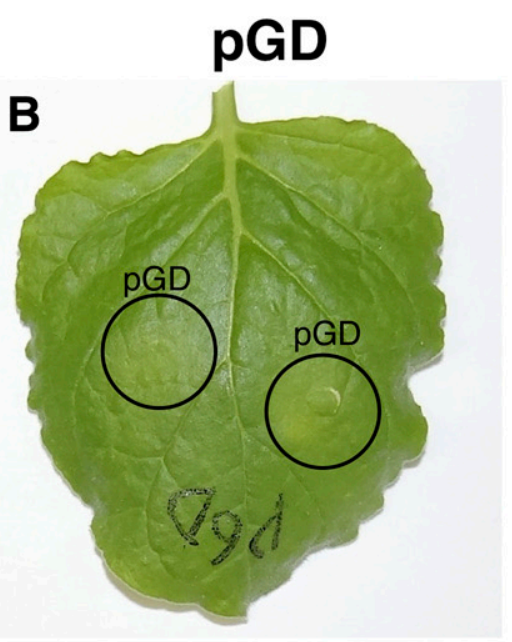

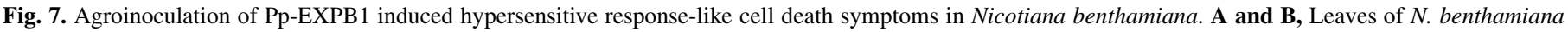

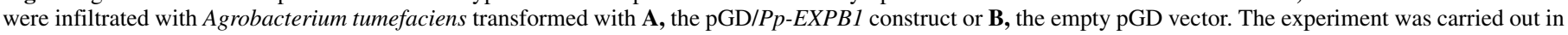

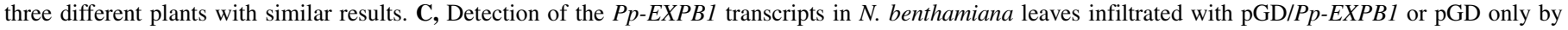
semiquantitative reverse transcription PCR. L1 and L2 correspond to the leaves from two independently inoculated plants. 
genes and the TMV resistance protein $\mathrm{N}$, along with the DEGs depicted by KEGG analysis may indicate that they are involved in the direct or indirect recognition of the Pp-EXPB1 effector protein and development of the HR-like symptoms on the leaves of PVX/ $P p-E X P B 1-i n f e c t e d$ and agroinoculated plants. Highly upregulated genes (Supplementary Table S4) included those encoding ethylene response factors (ERFs), which are key regulators in hormone and stress signaling (Müller and Munné-Bosch 2015); WRKY and NAC transcription factors, which are involved in coping with diverse biotic and abiotic stresses (Nuruzzaman et al. 2013; Pandey and Somssich 2009); and pathogenesis-related and LRR proteins, which are indispensable components of the plant immune system (Jones et al. 2016; Linthorst and Van Loon 1991). These results suggested that the expression of PVX/Pp-EXPB1 may also enhance the expression levels of a network of defense-related genes in $N$. benthamiana.
Confirmation of the transcriptomic data by qPCR analyses. To validate our RNA-seq data, the transcriptional levels of 21 arbitrarily selected DEGs were analyzed using qPCR (Table 2). From this set, 17 genes were up-regulated, and 4 genes were down-regulated. The qPCR data and the corresponding RNAseq values were consistent for the majority of the genes tested when leaves were challenged with PVX/Pp-EXPB1, whereas in the leaves inoculated with the genus Agrobacterium transformed with $P p$ $E X P B 1$, a reduced correlation ratio was observed (71\%).

\section{DISCUSSION}

Migratory RLNs affect host plants via mechanical stress induced by their movement and feeding and via the secretion of effector proteins, which are virulence factors that play important roles in different aspects of the disease development process. It is widely
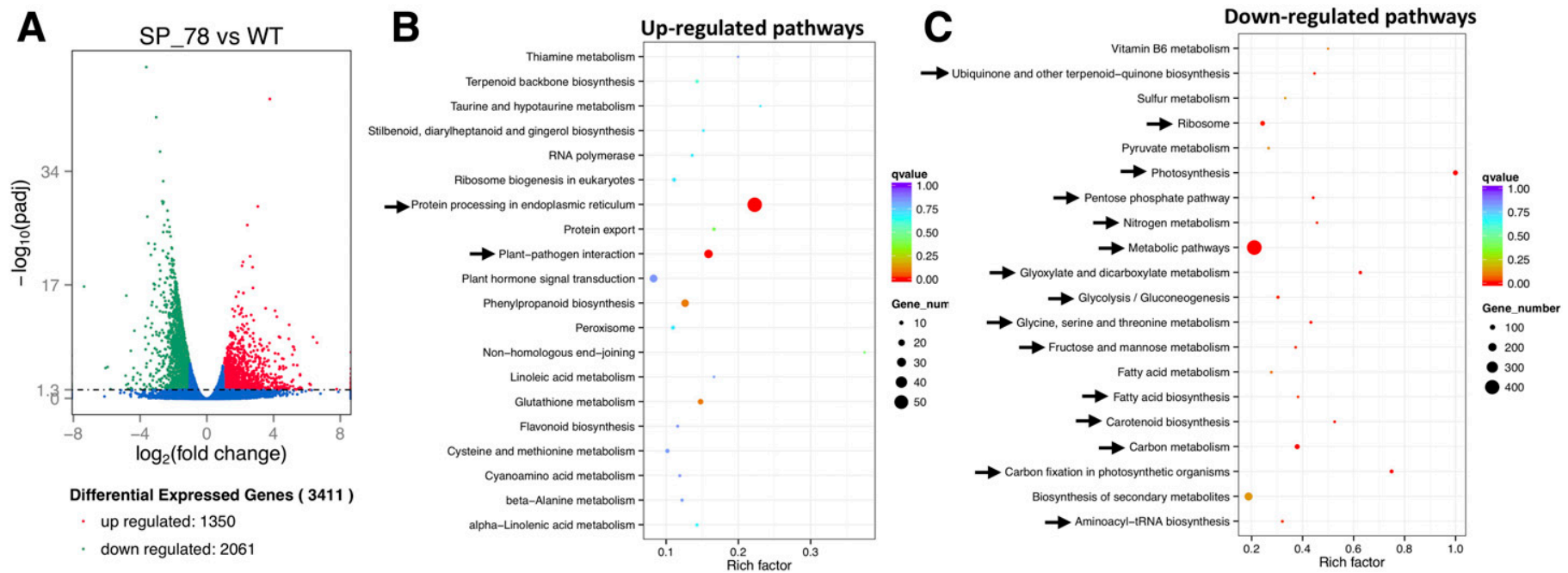

Fig. 8. Gene expression profiling in Nicotiana benthamiana plants inoculated with RNA transcripts derived from the PVX/Pp-EXPB1 and PVX-WT vectors. A, Volcano plot representation shows the total number of significant differentially expressed genes (DEGs) between PVX/Pp-EXPB1 and PVX-WT. B and C, Kyoto Encyclopedia of Genes and Genomes (KEGG) enrichment scatter plot analyses showing the total number of genes in the most represented pathways. The colored dots correspond to the $q$ value, and the dot size indicates the number of DEGs mapped to the reference pathways. Arrows indicate the significantly enriched KEGG pathways $(q$ values $<0.05)$.

TABLE 2. Validation of the RNA-sequencing (RNA-seq) data by quantitative real-time PCR

\begin{tabular}{|c|c|c|c|c|}
\hline Gene_id & BLAST Swiss-Prot & RNA-seq & PVX/Pp-EXPB 1 & $\begin{array}{l}\text { Agro pGD/ } \\
\text { Pp-EXPB1 }\end{array}$ \\
\hline Niben101Scf07491g00003.1 & P08252|CHI1_TOBAC Endochitinase A & 5.60 & 9.23 & 4.82 \\
\hline Niben101Scf09044g01012.1 & P14170|OSMO_TOBAC Osmotin OS & 5.36 & 9.66 & 3.58 \\
\hline Niben101Scf03069g01016.1 & Q6L406|R1B19_SOLDE Putative late blight resistance protein homolog R1B-19 & 4.67 & 4.64 & 1.60 \\
\hline Niben101Scf01015g01002.1 & P09762|WIN2_SOLTU Wound-induced protein WIN2 & 4.53 & 6.61 & 2.09 \\
\hline Niben101Scf02410g00002.1 & Q05537|CHID_SOLLC Basic endochitinase & 4.42 & 5.68 & 0.89 \\
\hline Niben101Scf04053g02006.1 & P11670|PRB1_TOBAC Basic form of pathogenesis-related protein 1 & 4.32 & 5.59 & 4.82 \\
\hline Niben101Scf06684g01005.1 & P51819|HSP83_IPONI Heat shock protein 83 & 3.78 & 4.97 & 1.15 \\
\hline Niben101Scf01398g00006.1 & Q6L403|R1B17_SOLDE Putative late blight resistance protein homolog R1B-17 & 3.60 & 4.17 & 3.75 \\
\hline Niben101Scf02041g00002.1 & P17514|CHIQ_TOBAC Acidic endochitinase Q & 3.42 & 3.99 & 1.45 \\
\hline Niben101Scf00975g01015.1 & $\begin{array}{l}\text { Q9FIZ3|GSO2_ARATH LRR receptor-like serine/threonine-protein kinase GSO2 } \\
\text { O }\end{array}$ & 3.38 & 3.18 & 1.09 \\
\hline Niben101Scf00107g03008.1 & P07053|PR1B_TOBAC Pathogenesis-related protein 1B & 3.25 & 2.66 & 1.72 \\
\hline Niben101Scf03925g01010.1 & Q9FIZ3|GSO2_ARATH LRR receptor-like serine/threonine-protein kinase GSO2 & 2.82 & 4.02 & 0.85 \\
\hline Niben101Scf06349g00008.1 & P27141|CAHC_TOBAC Carbonic anhydrase, chloroplastic & -3.53 & -4.26 & -1.61 \\
\hline Niben101Scf13600g01004.1 & P27492|CB21_TOBAC Chlorophyll a-b binding protein 16, chloroplastic & -3.67 & -4.09 & -1.82 \\
\hline
\end{tabular}


assumed that the aboveground symptoms of RLN infection originate from root damage rather than from the movement of nematode secretions to other parts of the plant and their toxicity (Davis and MacGuidwin 2000).

In this study, we confirmed the presence of four different expansin-like genes in $P$. penetrans, sequenced and characterized these genes, and demonstrated that the transient expression of one of the EXPNs, which was shown to be the most highly expressed during nematode infection, modulated immune responses in $N$. benthamiana plants.

Although three EXPNs consisted of an expansin domain coupled to a CBM2-like domain at the $\mathrm{N}$ terminus, a single member of this family, $P p-E X P B 1$, carried only an expansin domain. This gene structure is not unusual and follows a similar pattern found in other PPNs (Kikuchi et al. 2011). The exogenous expression of $\mathrm{Pp}$ $E X P B 1$ in the model species $N$. benthamiana caused a pleiotropic effect. Phenotypic abnormalities included severe dwarfing, chlorosis, HR-like lesions on leaves, and deteriorated roots and were accompanied by substantial underlying changes in gene expression patterns. Expansins are cell wall proteins that mediate cell wall loosening and cell enlargement and participate in many developmental processes where cell wall modifications occur: plant cell growth, fruit softening, abscission, emergence of root hairs, pollen tube invasion of the stigma, and style and meristem function (Sampedro and Cosgrove 2005). Expansins are present in all plants and some other organisms (Marowa et al. 2016). Speculatively, expansins secreted by RLNs during infection have the same function: that is, these expansins assist in nematode invasion and the subsequent migration of nematodes in the root tissue by cell wallloosening activities. Our results, however, indicate that the profound impact of the EXPN encoded by the $P p-E X P B 1$ gene on $N$. benthamiana plants is unlikely to be solely attributed to its cell wall relaxation ability and could also be attributed to other functions of the effector in disease development, such as interfering with the host immune response or effector-triggered immunity (ETI) (Jones and Dangl 2006).

Among the four genes that encode putative EXPNs in $P$. penetrans, $P p-E X P B 1$ was found to be the most highly upregulated during the interaction of the nematode with different host plants, which emphasizes its importance in nematode parasitism. The massive down-regulation of host genes involved in a broad range of biological processes may indicate the successful overtake of the host basal immune response and the promotion of effectorbased virulence. Thus, the basal response to Pp-EXPB1 in $N$. benthamiana plants is likely to be triggered by effectorassociated molecular patterns (EAMPs), implying a similar role of $P p-E X P B 1$ during the actual nematode infection. It cannot be excluded that the pathogenicity of $P p-E X P B 1$ might be associated with more diverse enzymatic activities than cell wall loosening, resembling those of the bacterial effectors (Abramovitch et al. 2006). For example, the glycoside hydrolase 12 protein XEG1 is required for Phytophthora sojae virulence, but it is also recognized as a pathogenesis-associated molecular pattern (PAMP) that triggers cell death and plant immunity (Ma et al. 2015).

Chloroplastic genes accounted for nearly $50 \%$ of all downregulated DEGs in PVX/Pp-EXPB1-infected plants. It was proposed in 2010 that a universal down-regulation of photosynthesisrelated gene expression constitutes an adaptive response to biotic stress by means of redirecting host resources to immediate defense needs (Bilgin et al. 2010). Indeed, coinciding with the suppression of chloroplastic DEGs, the PVX/Pp-EXPB1 plants exhibited widespread up-regulation of a network of defense-related genes. These genes included DEGs with potential roles in the recognition of $P p-E X P B 1$ and the advancement of disease resistance via ETI: two genes encoding putative homologs of the late blight resistance genes, R1B-19 and RB-17, and a homolog of the TMV-N. The broad up-regulation of DEGs implemented in the transcriptional control of stress-responsive genes-ERF, WRKY, and NAC-indicates that $P p-E X P B 1$ might be one of the core elements of the $P$. penetrans effectorome that not only targets important host molecular components to facilitate parasitism but also, elicits a major immune response (Vieira and Gleason 2019). The upregulation of the genes involved in protein processing in the ER emphasizes the role of the endoplasmic reticulum quality control (ERQC) mechanisms in the degradation of host proteins affected by the expression of $P p-E X P B 1$ in planta. The ERQC mechanisms are closely linked to calcium homeostasis (Araki and Nagata 2012), which may explain the up-regulation of several genes encoding calcium-binding proteins in $P p-E X P B 1$-expressing plants.

The agroinoculation experiments supported our hypothesis that $N$. benthamiana may possess an $R$ gene product specific for the recognition of Pp-EXPB1: agroinfiltration into $N$. benthamiana leaves caused a distinct HR response; however, this response was absent in the plants inoculated with the genus Agrobacterium transformed with the empty plasmid vector. Currently, several genes that confer resistant against PPNs have been identified (Williamson and Kumar 2006), most of them against root-knot or cyst nematodes. Although the exact identity of the putative $R$ gene for $P$. penetrans requires further investigation, orthologs of the potato late blight resistance genes $R 1 B-19$ and $R B-17$ may be among the candidates for this gene.

Overall, based on the biological experimentation and in silico analyses carried out in this work, we conclude that the $P$. penetrans gene $P p-E X P B 1$ encodes an EXPN that plays two major roles in nematode parasitism: (i) a virulence factor potentially involved in the recognition and modulation of the components of the plant immune system to advance nematode colonization and (ii) a protein that possibly mediates nematode movement and feeding by cell wall extension and loosening. During this interaction, in the plant, there seems to be a combination of EAMP-triggered immunity in the form of a basal response administered through the rearrangement of the plant defense recourses and possibly effector-triggered immunity (ETI), which is rendered by a plant genetic factor implicated in the resistance mechanisms against $P$. penetrans. If so, there could be a continuum between pattern-triggered immunity (PTI) and ETI-like responses to the candidate effector protein PpEXPB1, most likely because Pp-EXPB1 acts both as a PAMP activating PTI and as an effector that is recognized by the host NBLRR proteins (Jones and Dangl 2006; Thomma et al. 2011). Whether the proposed functions of the Pp-EXPB1 remain unchanged in the course of infection when a diverse array of nematode effectors is secreted into plant cells requires further investigation.

\section{LITERATURE CITED}

Abramovitch, R. B., Anderson, J. C., and Martin, G. B. 2006. Bacterial elicitation and evasion of plant innate immunity. Nat. Rev. Mol. Cell Biol. 7:601-611.

Ali, S., Magne, M., Chen, S., Côté, O., Stare, B. G., Obradovic, N., Jamshaid, L., Wang, X., Bélair, G., and Moffett, P. 2015. Analysis of putative apoplastic effectors from the nematode, Globodera rostochiensis, and identification of an expansin-like protein that can induce and suppress host defenses. PLoS One 10:e0115042.

Araki, K., and Nagata, K. 2012. Protein folding and quality control in the ER. Cold Spring Harb. Perspect. Biol. 4:a015438.

Bilgin, D. D., Zavala, J. A., Zhu, J., Clough, S. J., Ort, D. R., and DeLucia, E. H. 2010. Biotic stress globally downregulates photosynthesis genes. Plant Cell Environ. 33:1597-1613.

Bombarely, A., Rosli, H. G., Vrebalov, J., Moffett, P., Mueller, L. A., and Martin, G. B. 2012. A draft genome sequence of Nicotiana benthamiana to enhance molecular plant-microbe biology research.Mol. Plant-Microbe Interact. 25:1523-1530.

Boyes, D. C., Nam, J., and Dangl, J. L. 1998. The Arabidopsis thaliana RPM1 disease resistance gene product is a peripheral plasma membrane protein that is degraded coincident with the hypersensitive response. Proc. Natl. Acad. Sci. USA 95:15849-15854.

Brandizzi, F., Frigerio, L., Howell, S. H., and Schäfer, P. 2014. Endoplasmic reticulum-shape and function in stress translation. Front. Plant Sci. 5: 425 . 
Burke, M., Scholl, E. H., Bird, D. M., Schaff, J. E., Coleman, S., Crowell, R., Diener, S., Gordon, O., Graham, S., Wang, X., Windham, E., Wright, G. M., and Opperman, C. H. 2015. The plant parasite Pratylenchus coffeae carries a minimal nematode genome. Nematology 217:621-637.

Castillo, P., and Vovlas, N. 2007. Pratylenchus (Nematoda: Pratylenchidae): Diagnosis, Biology, Pathogenicity and Management, Nematology Monographs and Perspectives, Vol. 6. Brill, Leiden, The Netherlands.

Chen, C., Chen, Y., Jian, H., Yang, D., Dai, Y., Pan, L., Shi, F., Yang, S., and Liu, Q. 2018. Large-scale identification and characterization of Heterodera avenae putative effectors suppressing or inducing cell death in Nicotiana benthamiana. Front. Plant Sci. 8:2062.

Cosgrove, D. J. 2000. Loosening of plant cell walls by expansins. Nature 407: 321-326.

Danchin, E. G. J., Rosso, M.-N., Vieira, P., de Almeida-Engler, J., Coutinho, P. M., Henrissat, B., and Abad, P. 2010. Multiple lateral gene transfers and duplications have promoted plant parasitism ability in nematodes. Proc. Natl. Acad. Sci. USA 107:17651-17656.

Davis, E. L., and MacGuidwin, A. E. 2000. Lesion nematode disease. Plant Health Instructor. doi:

Denver, D. R., Brown, A. M. V., Howe, D. K., Peetz, A. B., and Zasada, I. A. 2016. Genome skimming: A rapid approach to gaining diverse biological insights into multicellular pathogens. PLoS Pathog 12:e1005713.

Edgar, R. C. 2004. MUSCLE: Multiple sequence alignment with high accuracy and high throughput. Nucleic Acids Res. 32:1792-1797.

Eves-van den Akker, S., Laetsch, D. R., Thorpe, P., Lilley, C. J., Danchin, E. G. D., Da Rocha, M., Rancurel, C., Holroyd, N. E., Cotton, J. A., Szitenberg, A., Grenier, E., Montarry, J., Mimee, B., Duceppe, M.-O., Boyes, I., Marvin, J. M. C., Jones, L. M., Yusup, H. B., Lafond-Lapalme, J., Esquibet, M., Sabeh, M., Rott, M., Overmars, H., Finkers-Tomczak, A., Smant, G., Koutsovoulos, G., Blok, V., Mantelin, S., Cock, P. J. A., Phillips, W., Henrissat, B., Urwin, P. E., Blaxter, M., and Jones, J. T. 2016. The genome of the yellow potato cyst nematode, Globodera rostochiensis, reveals insights into the basis of parasitism and virulence. Genome Biol. 17:124.

Fosu-Nyarko, J., and Jones, M. G. K. 2016. Advances in understanding the molecular mechanisms of root lesion nematode host interactions. Annu. Rev. Phytopathol. 54:253-278.

Goodin, M. M., Zaitlin, D., Naidu, R. A., and Lommel, S. A. 2008. Nicotiana benthamiana: Its history and future as a model for plant-pathogen interactions. Mol. Plant-Microbe Inter. 21:1015-1026.

Haegeman, A., Joseph, S., and Gheysen, G. 2011. Analysis of the transcriptome of the root lesion nematode Pratylenchus coffeae generated by 454 sequencing technology. Mol. Biochem. Parasitol. 178:7-14.

Jones, J. D., Vance, R. E., and Dangl, J. L. 2016. Intracellular innate immune surveillance devices in plants and animals. Science 354:aaf6395.

Jones, J. D. G., and Dangl, J. L. 2006. The plant immune system. Nature 444: 323-329.

Jones, J. T., Haegeman, A., Danchin, E. G., Gaur, H. S., Helder, J., Jones, M. G., Kikuchi, T., Manzanilla-López, R., Palomares-Rius, J. E., Wesemael, W. M., and Perry, R. N. 2013. Top 10 plant-parasitic nematodes in molecular plant pathology. Mol. Plant Pathol. 14:946-961.

Kikuchi, T., Cotton, J. A., Dalzell, J. J., Hasegawa, K., Kanzaki, N., McVeigh, P., Takanashi, T., Tsai, I., Assefa, S. A., Cock, P. J. A., Dan Otto, T., Hunt, M., Reid, A. J., Sanchez-Flores, A., Tsuchihara, K., Yokoi, T., Larsson, M. C., Miwa, J., Maule, A. G., Sahashi, N., Jones, J. T., and Berriman, M. 2011. Genomic insights into the origin of parasitism in the emerging plant pathogen Bursaphelenchus xylophilus. PLoS Pathog 7:e1002219.

Koch, E., and Slusarenko, A. 1990. Arabidopsis is susceptible to infection by a downy mildew fungus. Plant Cell 2:437-445.

Kørner, C. J., Du, X., Vollmer, M. E., and Pajerowska-Mukhtar, K. M. 2015. Endoplasmic reticulum stress signaling in plant immunity - at the crossroad of life and death. Int. J. Mol. Sci. 16:26582-26598.

Kudla, U., Qin, L., Milac, A., Kielak, A., Maissen, C., Overmars, H., Popeijus, H., Roze, E., Petrescu, A., Smant, G., Bakker, J., and Helder, J. 2005. Origin, distribution and 3D-modeling of Gr-EXPB1, an expansin from the potato cyst nematode Globodera rostochiensis. FEBS Lett. 579:2451-2457.

Lacomme, C., and Chapman, S. 2008. Use of potato virus X (PVX)-based vectors for gene expression and virus-induced gene silencing (VIGS). Curr. Protoc. Microbiol. 8:16I.1.1-16I.1.13.

Linthorst, H. J. M., and Van Loon, L. C. 1991. Pathogenesis-related proteins of plants. Crit. Rev. Plant Sci. 10:123-150.

Liu, D., Shi, L., Han, C., Yu, J., Li, D., and Zhang, Y. 2012. Validation of reference genes for gene expression studies in virus-infected Nicotiana benthamiana using quantitative real-time PCR. PLoS One 7:e46451.

Liu, J., Peng, H., Cui, J., Huang, W., Kong, L. J., Clarke, L., Jian, H., Wang, G. L., and Peng, D. 2016. Molecular characterization of a novel effector expansin-like protein from Heterodera avenae that induces cell death in Nicotiana benthamiana. Sci. Rep. 6:35677.
Livak, K. J., and Schmittgen, T. D. 2001. Analysis of relative gene expression data using real-time quantitative PCR and the 2(-Delta $\mathrm{C}(\mathrm{T})$ ) method. Methods 25:402-408.

Ma, Z., Song, T., Zhu, L., Ye, W., Wang, Y., Shao, Y., Dong, S., Zhang, Z., Dou, D., Zheng, X., Tyler, B. M., and Wang, Y. 2015. A Phytophthora sojae glycoside hydrolase 12 protein is a major virulence factor during soybean infection and is recognized as a PAMP. Plant Cell 27: 2057-2072.

Marowa, P., Ding, A., and Kong, Y. 2016. Expansins: Roles in plant growth and potential applications in crop improvement. Plant Cell Rep. 35: 949-965.

Müller, M., and Munné-Bosch, S. 2015. Ethylene response factors: A key regulatory hub in hormone and stress signaling. Plant Physiol. 169:32-41.

Nemchinov, L. G., and Natilla, A. T. 2007. Transient expression of the ectodomain of matrix protein 2 (M2e) of avian influenza A virus in plants. Protein Expr. Purif. 56:153-159.

Nicol, P., Gill, R., Fosu-Nyarko, J., and Jones, M. G. K. 2012. De novo analysis and functional classification of the transcriptome of the root lesion nematode, Pratylenchus thornei, after 454 GS FLX sequencing. Int. J. Parasitol. 42:225-237.

Nuruzzaman, M., Sharoni, A. M., and Kikuchi, S. 2013. Roles of NAC transcription factors in the regulation of biotic and abiotic stress responses in plants. Front. Microbiol. 4:248.

Opperman, C. H., Bird, D. M., Williamson, V. M., Rokhsar, D. S., Burke, M., Cohn, J., Cromer, J., Diener, S., Gajan, J., Graham, S., Houfek, T. D., Liu, Q., Mitros, T., Schaff, J., Schaffer, R., Scholl, E., Sosinski, B. R., Thomas, V. P., and Windham, E. 2008. Sequence and genetic map of Meloidogyne hapla: A compact nematode genome for plant parasitism. Proc. Natl. Acad. Sci. USA 105:14802-14807.

Pandey, S. P., and Somssich, I. E. 2009. The role of WRKY transcription factors in plant immunity. Plant Physiol. 150:1648-1655.

Peng, H., Gao, B. L., Kong, L. A., Yu, Q., Huang, W. K., He, X. F., Long, H. B., and Peng, D. L. 2013. Exploring the host parasitism of the migratory plant-parasitic nematode Ditylenchus destuctor by expressed sequence tags analysis. PLoS One 8:e69579.

Petersen, T. N., Brunak, S., Von Heijne, G., and Nielsen, H. 2011. SignalP 4.0: Discriminating signal peptides from transmembrane regions. Nat. Methods 8:785-786.

Qin, L., Kudla, U., Roze, E. H., Goverse, A., Popeijus, H., Nieuwland, J., Overmars, H., Jones, J. T., Schots, A., Smant, G., Bakker, J., and Helder, J. 2004. A nematode expansin acting on plants. Nature 427:30.

Sampedro, J., and Cosgrove, D. J. 2005. The expansin superfamily. Genome Biol. 6:242.

Smant, G., Stokkermans, J., Yan, Y., de Boer, J. M., Baum, T., Wang, X., Hussey, R. S., Davis, E. L., Gommers, F. J., Henrissat, B., Helder, J., Schots, A., and Bakker, J. 1998. Endogenous cellulases in animals: Isolation of $\beta$-1,4-endoglucanase genes from two species of plant-parasitic cyst nematodes. Proc. Natl. Acad. Sci. USA 95:4906-4911.

Tanabe, A. S. 2011. Kakusan and Aminosan: Two programs for comparing nonpartitioned, proportional and separate models for combined molecular phylogenetic analysis of multilocus sequence data. Mol. Ecol. Resour. 11: 914-921.

Thomma, B. P., Nürnberger, T., and Joosten, M. H. 2011. Of PAMPs and effectors: The blurred PTI-ETI dichotomy. Plant Cell 23:4-15.

Vicente, C. S. L., Nemchinov, L. G., Mota, M., Eisenback, J. D., Kamo, K., and Vieira, P. 2019. Identification and characterization of the first pectin methylesterase gene discovered in the root lesion nematode Pratylenchus penetrans. PLoS One 14:e0212540.

Vieira, P., Eves-van den Akker, S., Verma, R., Wantoch, S., Eisenback, J. D., and Kamo, K. 2015. The Pratylenchus penetrans transcriptome as a source for the development of alternative control strategies: Mining for putative genes involved in parasitism and evaluation of in planta RNAi. PLoS One 10:e0144674.

Vieira, P., and Gleason, C. 2019. Plant-parasitic nematode effectors-insights into their diversity and new tools for their identification. Curr. Opin. Plant Biol. 50:37-43.

Vieira, P., Maier, T. R., Eves-van den Akker, S., Howe, D. K., Zasada, I., Baum, T. J., Eisenback, J. D., and Kamo, K. 2018. Identification of candidate effector genes of Pratylenchus penetrans. Mol. Plant Pathol. 19:1887-1907.

Vieira, P., Mowery, J., Eisenback, J. D., Shao, J., and Nemchinov, L. G. 2019. Cellular and transcriptional responses of resistant and susceptible cultivars of alfalfa to the root lesion nematode, Pratylenchus penetrans. Front. Plant Sci. 10:971.

Williamson, V. M., and Kumar, A. 2006. Nematode resistance in plants: The battle underground. Trends Genet. 22:396-403.

Zunke, U. 1990. Observations on the invasion and endoparasitic behavior of the root lesion nematode Pratylenchus penetrans. J. Nematol. 22: 309-320. 